\title{
Emotion Detection from text: A Survey
}

\author{
Lea Canales, Patricio Martínez-Barco \\ Department of Software and Computing Systems \\ University of Alicante \\ \{lcanales,patricio\}@dlsi.ua.es
}

\begin{abstract}
This survey describes recent works in the field of Emotion Detection from text, being a part of the broader area of Affective Computing. This survey has been inspired on the well-known fact that, despite there is a lot of work on emotional detection systems, a lot of work is expected to be done yet. The increment of these systems is due to the large amount of emotional data available in Social Web. Detecting emotions from text have attracted the attention of many researchers in computational linguistics because it has a wide range of applications, such as suicide prevention or measuring well-being of a community. This paper mainly collects works based on lexical and machine learning approaches and these works are classificated in accordance with the emotional model and the approach used.
\end{abstract}

\section{Introduction}

This survey describes recent works in the field of emotion or affect detection from text. Emotion detection is part of the broader area of Affective Computing with aims to enable computers recognize and express emotions [Picard 1997]. Current affect detection systems are with respect to individual modalities or channels, such as face, voice and text [Calvo 2010]. In this survey, we have focused on reviewing works about emotion detection from text.

Emotion detection and analysis has been widely researched in neuroscience, psychology and behavior science, as they are an important element of human nature. In computer science, this task has also attracted the attention of many researchers, especially in the field of human computer interactions [Strapparava 2008].

In computational linguistics, the detection of emotion states of a person by analyzing a text document written by him/her can have many applications in different fields, such as in e-learning environment [Rodriguez 2012] or suicide prevention [Desmet 2013, Vaassen 2014]. For this reason, we decided to develop a survey about emotion detection systems from text and make it available to researcher community.

In this survey, we classify the most relevant emotion detection works in accordance with the emotional model and the approach used. A numerical comparison is not possible since each work used different data sets to evaluate their systems.

Regarding the search strategy used in the survey, we have looked for all of papers related to emotion detection from text in different research databases like Scopus ${ }^{1}$ or IEEE Xplore ${ }^{2}$. Later on, we have reviewed the papers obtained of these databases and have selected the best papers that use lexical approach or machine learning approach in their emotion detection systems. The selection criterion used is based on the relevance of each work in the field of Affective Computing.

This paper is organized as follows. In section 2, describes the emotional models. Section 3, the different computational

\footnotetext{
1 http://www.scopus.com/

2 http://ieeexplore.ieee.org/
} 
approaches for emotion detection is described. Finally, in section 4, we express our conclusions about this survey.

\section{Emotion models}

When emotional detection systems are analyzed, it is important to focus our interest on describing and explaining how the emotion models are established, as they are, the basis of these systems.

According to research in psychology, there is a number of theories about how to represent emotions [Cowie 2003] but two are the most important and the most often used in existing approaches in Sentiment Analysis [Francisco 2013]: emotional categories and emotional dimensions.

Emotional categories approaches are focused on model emotions based on distinct emotion classes or labels. The categorical model assumes that there are discrete emotion categories. The Ekman's basic emotion model is within this approach. [Ekman 1999] concluded that the six basic emotions are ANGER, DISGUST, FEAR, HAPPINESS, SADNESS and SURPRISE. [Plutchik 1980] define a set of eight basic bipolar emotions, consisting of a superset of Ekman's and with two additions: TRUST and AnTICIPATION. These eight emotions are organized into four bipolar sets: joy vs. sadness, anger vs. fear, trust vs. disgust, and surprise vs. anticipation.

Emotional dimensions approaches represent affects in a dimensional form. Each emotion occupies a location in this space [Kim 2011]. One of the more representative model of these approaches is [Russell 1980]. Rusell's Circumplex Model of Affect suggests that emotions are distributed in a two-dimensional circular space: valence dimension and arousal dimension, as show Figure 1. The valence dimension indicates how much PLEASANT and UNPLEASANT is an emotion. The arousal dimension differentiates ACTIVATION and DEACTIVATION states. In this approach, we also find the Mehrabian's model, a model based on a three-dimensional PAD (Pleasure - Arousal - Dominance) representation [Mehrabian 1996]. The dominance dimension indicates whether the subject feels in control of the situation or not.

Although existing emotional categories and emotional dimensions for representing affective states, categorical approaches are the most commonly used [Calvo 2013], as we can check out in next section. Most of computational approaches are based on emotional categories, due to its simplicity and familiarity. Nevertheless, emotional categories may not cover all emotions adequately because emotion categories are limited. This is a major benefit of emotional dimensional models. They are not correlated to a certain emotional state and are able to capture subtle emotion concepts that differ only slightly. In addition, a dimensional emotion model provides a means for measuring similarity between affective states [Kim 2011].

As we can observe, there are not an emotion model better than other. Both models have advantages and disadvantages. The election of an emotion model depends on the set of emotions that we want detect.

\section{Computational approaches for emotion detection}

Emotion detection techniques can be divided into lexicon based approaches and machine learning approaches. On the one hand, lexicon based approaches rely on lexical resources such as lexicons, bags of words or ontologies. On the other hand, Machine Learning (ML) approaches apply 
ML algorithms based on linguistic features.

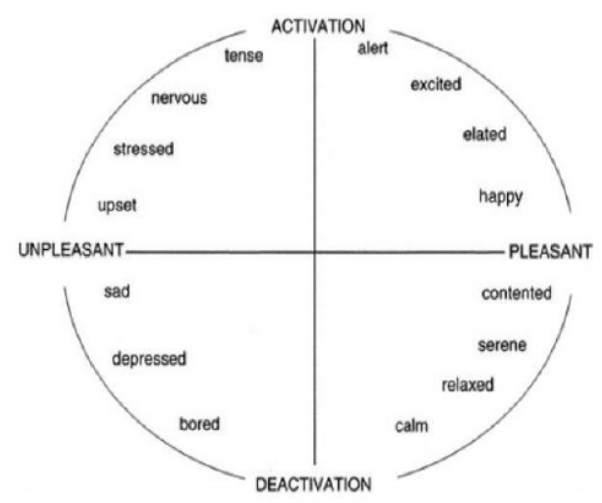

Figure 1: Graphical representation of the Circumplex Model of Affect.

\subsection{Lexicon-based approaches}

Lexicon based approaches are approaches that only use one or several lexical resources to detect emotions detection.

Among these approaches, we can find keyword-based approaches that are based on predetermining a set of terms to classify the text into emotion categories. In [Strapparava 2008], as a baseline, they implemented a simple algorithm that checked the presence of affective words in the headlines, and computed a score that reflected the frequency of the words in this affective lexicon in the text. They used WordNet-Affect [Strapparava 2004].

Also among Lexicon based approaches, we find the ontology-based ones. [Balahur 2011] use EmotiNet - a resource for the detection of emotion from text based on commonsense knowledge on concepts, their interaction and their affective consequence - to detect emotion. EmotiNet models situations as chains of actions and their corresponding emotional effect using an ontological representation. Their evaluation consists in testing if by employing the model they build and the knowledge contained in the core of EmotiNet, they are able to detect the emotion expressed in new examples pertaining to the categories in International Survey of Emotional
Antecedents and Reactions (ISEAR), through computing the similarity between the emotion chain of the new situation and the EmotiNet emotion chains. Their evaluation shows that the structure and content of EmotiNet are appropriate to address the automatic treatment of implicitly expressed affect. [Sykora 2013] also use an ontology approach to solve the problem of fine-grained emotion detection in text. Their approach detects a range of eight high-level emotions; anger, confusion, disgust, fear, happiness, sadness, shame and surprise.

Statistical approach is also considered as a Lexical approach. Most knowledge-based works use Latent Semantic Analysis (LSA), a statistical approach for analyzing the relationships between a set of documents and the terms mentioned in these documents in order to produce a set of meaningful patterns related to the documents and terms [Deerwester 1999]. [Gill 2008] used LSA and the Hyperspace Analogue to Language (HAL) to automatically compute the semantic similarity between the texts and emotions keywords. Recently, [Wang 2013] propose a method that uses an improved LSA algorithm for text emotion classification on ISEAR dataset.

\subsection{Machine Learning-based} approaches

Machine learning is a scientific discipline that deals with the construction and study of algorithms that can learn from data [Kovahi 1998]. Such algorithms operate by building a model based on inputs and using these inputs to make predictions or decisions, rather than following only explicitly programmed instructions [Bishop 2006].

Specifically in emotion detection, Machine learning algorithms are used to learn how detect emotions. These 
approaches can be divided into supervised and unsupervised learning.

Supervised learning approaches rely on a labelled training data, a set of training examples. The supervised learning algorithm analyses the training data and infers a function, which we use for mapping new examples [Mohri 2012].

A labelled corpus is a large and structured set of text that it is necessary annotated with emotional tags. In this case, the annotation process is considered as one of their most important disadvantages as it becomes a tedious and time-consuming task. However, there are recent works related with emotion detection in Twitter messages, where the training examples are automatically labelled through hashtags and emoticons contained. [Hasan 2014, Wang 2012, Roberts 2012, Suttles 2013] among others, are proposals that use this method for labeling training data automatically. Moreover, [Hasan 2014a] confirms that hashtags are indeed good emotion labels.

Concerning works that apply supervised learning algorithms, we can find both the categorical and the dimensional approaches to base their emotional models. Categorical approaches are the most commonly used in emotion detection [Calvo 2013]. One of the first works based in this model is [Alm 2005]. This proposal presented an empirical study of applying supervised machine learning with the SNoW learning architecture [Roth 1999]. They used an annotated corpus with an extended set of Ekman basic emotions. [Strapparava 2008], in one of the experiment presented in their work, applied Naïve Bayes classifier trained on the blog entries from LiveJournal.com ${ }^{3}$. They used a collection of blogposts annotated with Ekman's emotions. More recently, [Balabantaray 2012] presents an Emotion classifier that is able to determinate the emotion class of the person writing. Their emotion classifier is

\footnotetext{
3 http://www.livejournal.com/
}

based on multi-class SVM kernels and takes decisions according to the basic emotions identified by Ekman [Ekman 1999]. [Roberts 2012] also use the Ekman's six basic emotions and include LOVE emotion. Their system uses a series of binary SVM classifiers to detect each of the seven emotions. Other related work with categorical emotion models, [Suttles 2013] classify emotions according to a set of eight basic bipolar emotions defined by Plutchick. This allows them to treat the multi-class problem of emotion classification as a binary problem for opposing emotion pairs. Their approach applies Distant Supervision [Mintz 2009].

About works that apply supervised learning approach and use dimensional emotion model, we can find the work of [Hasan 2014], where they propose an approach for automatically classifying text messages of individual to infer their emotional states. They use the Rusell's Circumplex Model of Affect as emotion model and train supervised classifiers to detect multiple emotion. Specifically, they have compared the accuracy of SVM, KNN, Decision Tree and Naïve Bayes for classifying Twitter messages.

Regarding unsupervised learning approaches, these algorithms try to find hidden structure in unlabeled data in order to build models for emotion classification [Mohri 2012].

As occurs in supervised learning, among unsupervised learning proposals also it can be found systems based on categorical and dimensional emotion models.

With respect to works based in categorical emotion model, [Strapparava 2008] apply unsupervised techniques combining LSA with WordNet Affect [Strapparava 2004]. This proposal used the Ekman's basic emotions. [Agrawal 2012] proposes a novel unsupervised context-based approach based on a methodology that does not depend on any 
existing affect lexicon, thereby their model is flexible enough to classify sentences beyond Ekman's model of six basic emotions. [Calvo 2013] presents different categorical approaches based on Vector Space Model (VSM) with three dimensionality reduction techniques: Latent Semantic Analysis (LSA), Probabilistic Latent Semantic Analysis (PLSA) and Non-negative Matrix Factorization (NMF). This work conclude that NMF-based categorical classification performs the best among categorical approaches to classification.

About unsupervised approach with dimensional emotion model, we find [Calvo 2013]. This work used a normative database ANEW [Bradley 1999] to produce tree-dimensional vectors (valence, arousal, dominance) for each pseudo-document.

The articles presented in this survey are summarized in Table 1.

\section{Conclusion}

In this survey, we have started discussing the emotion models defined by psychologies because it is the base of emotion detection. As concluding by [Calvo 2013] and we have check out, categorical approach is the model more used in emotion detection systems.

Regarding Lexical approaches, keyword-based approaches are easily implementable and we can obtain good accuracy values, even though this approach has drawbacks: determining the content of the emotion lexicon is subjective, obtaining wrong recall values and the select words may be ambiguous [Suttles 2013]. Moreover, it is not suitable for wide range of domains.

With respect to approaches based on ontologies let us use commonsense knowledge and improve recall values but the creation of an emotional ontology is a tedious and time-consuming task.

Consequently, lexical resources usually are used as features in Machine Learning algorithms.

As for Machine Learning approaches, the supervised learning approach is more used in emotion detection because it usually leads to better results than unsupervised learning [Kim 2011]. Although, these approaches need labelling training examples and annotating of examples, which is a time-consuming task. For this reason, several researches have analyzed as 


\begin{tabular}{|c|c|c|c|}
\hline Papers & Categories & Emotion Model & Approaches \\
\hline $\begin{array}{l}\text { (Strapparava and } \\
\text { Mihalcea, 2008) }\end{array}$ & $\begin{array}{l}\text { Anger, Disgust, Fear, Joy, Sadness, } \\
\text { Surprise }\end{array}$ & Categorical & Lexical-based \\
\hline (Gill et al., 2008) & $\begin{array}{l}\text { Anger, Fear, Surprise, Joy, Anticipa- } \\
\text { tion, Acceptance, Sadness, Disgust }\end{array}$ & Categorical & Lexical-based \\
\hline $\begin{array}{l}\text { (Balahur et al., } \\
\text { 2011) }\end{array}$ & $\begin{array}{l}\text { Anger, Disgust, Fear, Guilt, Joy, Sad- } \\
\text { ness, Shame }\end{array}$ & Categorical & Lexical-based \\
\hline $\begin{array}{l}\text { (Sykora } \text { et al., } \\
2013 \text { ) }\end{array}$ & $\begin{array}{l}\text { Anger, Confusion, Disgust, Fear, } \\
\text { Happiness, Sadness, Shame, Surprise }\end{array}$ & Categorical & Lexical-based \\
\hline $\begin{array}{l}\text { (Wang and Zheng, } \\
\text { 2013) }\end{array}$ & $\begin{array}{l}\text { Anger, Disgust, Fear, Guilt, Joy, Sad- } \\
\text { ness, Shame }\end{array}$ & Categorical & Lexical-based \\
\hline (Alm et al., 2005) & $\begin{array}{l}\text { Anger, Disgust, Fear, Happiness, } \\
\text { Sadness, Positively Surprise, Nega- } \\
\text { tively Suprise }\end{array}$ & Categorical & $\begin{array}{l}\text { Supervised } \\
\text { Learning-based }\end{array}$ \\
\hline $\begin{array}{l}\text { (Strapparava and } \\
\text { Mihalcea, 2008) }\end{array}$ & $\begin{array}{l}\text { Anger, Disgust, Fear, Joy, Sadness, } \\
\text { Surprise }\end{array}$ & Categorical & $\begin{array}{l}\text { Supervised } \\
\text { Learning-based }\end{array}$ \\
\hline $\begin{array}{l}\text { (Balabantaray } \text { et } \\
\text { al., 2012) }\end{array}$ & $\begin{array}{l}\text { Anger, Disgust, Fear, Happiness, } \\
\text { Sadness, Surprise }\end{array}$ & Categorical & $\begin{array}{l}\text { Supervised } \\
\text { Learning-based }\end{array}$ \\
\hline $\begin{array}{l}\text { (Roberts et al., } \\
2012)\end{array}$ & $\begin{array}{l}\text { Anger, Disgust, Fear, Joy, Sadness, } \\
\text { Surprise, Love }\end{array}$ & Categorical & $\begin{array}{l}\text { Supervised } \\
\text { Learning-based }\end{array}$ \\
\hline $\begin{array}{l}\text { (Suttles and Ide, } \\
\text { 2013) }\end{array}$ & $\begin{array}{l}\text { Anger, Disgust, Fear, Happiness, } \\
\text { Sadness, Surprise, Trust, Anticipa- } \\
\text { tion }\end{array}$ & Categorical & $\begin{array}{l}\text { Supervised } \\
\text { Learning-based }\end{array}$ \\
\hline $\begin{array}{l}\text { (Hasan et al., } \\
\text { 2014b) }\end{array}$ & $\begin{array}{l}\text { Happy-Active, Happy-Inactive, } \\
\text { Unhappy-Active, Unhappy-Inactive }\end{array}$ & Dimensional & $\begin{array}{l}\text { Supervised } \\
\text { Learning-based }\end{array}$ \\
\hline $\begin{array}{l}\text { (Strapparava and } \\
\text { Mihalcea, 2008) }\end{array}$ & $\begin{array}{l}\text { Anger, Disgust, Fear, Joy, Sadness, } \\
\text { Surprise }\end{array}$ & Categorical & $\begin{array}{l}\text { Unsupervised } \\
\text { Learning-based }\end{array}$ \\
\hline $\begin{array}{l}\text { (Agrawal and An, } \\
\text { 2012) }\end{array}$ & $\begin{array}{l}\text { Anger, Disgust, Fear, Happiness, } \\
\text { Sadness, Surprise }\end{array}$ & Categorical & $\begin{array}{l}\text { Unsupervised } \\
\text { Learning-based }\end{array}$ \\
\hline $\begin{array}{l}\text { (Calvo and Kim, } \\
\text { 2013) }\end{array}$ & Anger-Disgust, Fear, Joy, Sadness & Categorical & $\begin{array}{l}\text { Unsupervised } \\
\text { Learning-based }\end{array}$ \\
\hline $\begin{array}{l}\text { (Calvo and Kim, } \\
\text { 2013) }\end{array}$ & Anger-Disgust, Fear, Joy, Sadness & Dimensional & $\begin{array}{l}\text { Unsupervised } \\
\text { Learning-based }\end{array}$ \\
\hline
\end{tabular}

Table 1: Emotion Detection approaches

realize this task automatically and when our system process Twitter messages, the messages can be annotated through hashtags or emotions that it contains.

Although unsupervised learning approach leads worse results than supervised learning, it can be a good election for the emotion detection task because the emotional interpretations of a text can be highly subjective and the annotation task is an error prone task [Kim 2011].

In conclusion, Machine Learning approaches are better option for detection emotion task since we obtain a model is also able to detect emotions in texts that have only an indirect reference to an emotions. Although, it is important use a good lexical resource as features in
Machine Learning algorithms to obtain good results.

Concerning pending tasks in emotion detection field, we consider really important that researcher community establish an annotated corpus and a set of metrics that it may be used to evaluate the different existing systems and the future systems. Moreover, in emotional detection systems based on machine learning approach, we have detected that most of these systems use features based on a shallow analysis on the text as: n-grams, punctuation, emoticons or Part-Of-Speech. Hence, we propose a new direction focuses on deep analysis, since we consider that if we use features based on a deep analysis on the text we could improve the emotional detection systems. 
Acknowledgments

This research has been supported by the FPI grant (BES-2013-065950) from the Spanish Ministry of Science and Innovation, under the project LEGOLANGUAGE (TIN2012-31224) funded by the Spanish Government. It has been also funded by the Valencian Government (grant no. PROMETEOII/2014/001).

\section{References}

Ameeta Agrawal and Aijun An. 2012. Unsupervised Emotion Detection from Text Using Semantic and Syntactic Relations. In 2012 IEEE/WIC/ACM International Conferences on Web Intelligence and Intelligent Agent Technology, pages 346-353. IEEE Computer Society, December.

Cecilia Ovesdotter Alm, Dan Roth, and RichardSproat. 2005. Emotions from text: machine learning for text-based emotion prediction. Proc. Conf. Human Language Technology and Empirical Methods in Natural Language Processing, pages 579-586.

R C Balabantaray, Mudasir Mohammad, and Nibha Sharma. 2012. Multi-Class Twitter Emotion Classification: A New Approach. International Journal of Applied Information Systems (IJAIS), 4(1):48-53.

Alexandra Balahur, Jesús M. Hermida, and Andrés Montoyo. 2011. Detecting Implicit Expressions of Sentiment in Text Based on Commonsense Knowledge. In 2nd Workshop on Computational Approaches to Subjectivity and Sentiment Analysis, ACL-HLT 2011, pages 53-60.

C. M. Bishop. 2006. Pattern Recognition and Machine Learning. Springer. Margaret M Bradley and Peter J Lang. 1999. Affective Norms for English Words (ANEW): Instruction Manual and Affective Ratings. Technical report, The Center for Research in Psychophysiology, University of Florida.
Rafael A Calvo and Sunghwan Mac Kim. 2013. Emotions in text: dimensional and categorical models. Computational Intelligence, 29(3).

Rafael A Calvo and Senior Member. 2010. Affect Detection: An Interdisciplinary Review of Models, Methods, and Their Applications. IEEE Transactions on Affective Computing, 1(1):18-37.

Roddy Cowie and Randolph R. Cornelius. 2003. Describing the emotional states that are expressed in speech. Speech Communication, 40(1-2):5-32, April.

Scott Deerwester, Susan T. Dumais, George W. Furnas, Thomas K. Landauer, and Richard Harshman.1999. Indexing by latent semantic analysis. Journal of the American Society for Information Science, 41(6):391-407, September.

Bart Desmet and Véronique Hoste. 2013. Emotion detection in suicide notes. Expert Systems with Applications, 40(16):6351-6358, November.

Paul Ekman. 1999. Basic emotions. In Handbook of cognition and emotion, pages 45-60. Virginia Francisco and Pablo Gerv'as. 2013. EmoTag: An Approach to Automated Mark-Up of Emotions in Texts. Computational Intelligence, 29(4):680-721.

Alastair J. Gill, Robert M. French, Darren Gergle, and Jon Oberlander. 2008. Identifying Emotional Characteristics from Short Blog Texts. In 30th Annual Meeting of the Cognitive Science Society, pages 2237-2242.

Maryam Hasan, Emmanuel Agu, and Elke Rundensteiner. 2014a. Using Hashtags as Labels for Supervised Learning of Emotions in Twitter Messages.

Maryam Hasan, Elke Rundensteiner, and Emmanuel Agu. 2014b. EMOTEX: Detecting Emotions in Twitter Messages. In ASE BIGDATA/SOCIALCOM/CYBERSECURI TY Conference, pages 27-31.

Sunghwan Mac Kim. 2011. Recognising Emotions and Sentiments in Text. Ph.D. thesis, University of Sydney. 
Ron Kovahi and Foster Provost. 1998. Glossary of terms. Machine Learning, pages 271-274.

A.

Mehrabian.

1996.

Pleasure-arousal-dominance: A general framework for describing and measuring individual.

Current Psychology, 15(4):505-525. M. Mintz, S. Bills, R. Snow, and D. Jurafsky. 2009. Distant supervision for relation extraction without labeled data. In Proceedings of the Joint Conference of the 47th Annual Meeting of the ACL and the 4th International Joint Conference on Natural Language Processing of the AFNLP, pages 1003-1011.

Mehryar Mohri, Afshin Rostamizadeh, and Ameet Talwalkar. 2012. Foundations of Machine Learning. MIT Press.

Rosalind W. Picard. 1997. Affective computing. MIT Press Cambridge, MA, USAc 1997. R. Plutchik. 1980. Emotion: Theory, Research and Experience. In Theories of emotion, volume 11, page 399. Academic Press.

Kirk Roberts, Michael A Roach, Joseph Johnson, Josh Guthrie, and Sanda M Harabagiu. 2012. EmpaTweet: Annotating and Detecting Emotions on Twitter. In Nicoletta Calzolari (Conference Chair) Piperidis, Khalid Choukri, Thierry Declerck, Mehmet U־gur Do־gan, Bente Maegaard, Joseph Mariani, Asuncion Moreno, Jan Odijk, and Stelios, editors, Proceedings of the Eight International Conference on Language Resources and Evaluation (LREC'12). European Language Resources Association (ELRA).

Pilar Rodriguez, Alvaro Ortigosa, and Rosa M. Carro. 2012. Extracting Emotions from Texts in ELearning Environments. In 2012 Sixth International Conference on Complex, Intelligent, and Software Intensive Systems, pages $887-892$. Ieee, July.

Dan Roth, Chad Cumby, Andy Carlson, and Jeff Rosen. 1999. The SNoW Learning Architecture. Technical report, UIUC Computer Science Department. J.A. Russell. 1980. A circumplex model of affect. Journal of Personality and Social Psychology, 39(6):1161-1178.

Carlo Strapparava and Rada Mihalcea. 2008.
Learning to identify emotions in text. In Proceedings of the 2008 ACM symposium on Applied computing - SAC '08, pages 1556-1560, New York, New York, USA. ACM Press.

Carlo Strapparava and Alessandro Valitutti. 2004. WordNet-Affect: an Affective Extension of Word-Net. In 4th International Conference on Language Resources and Evaluation, pages 1083-1086.

Jared Suttles and Nancy Ide. 2013. Distant Supervision for Emotion Classification with Discrete Binary Values. In Alexander Gelbukh, editor, Computational Linguistics and Intelligent Text Processing, volume 7817 of Lecture Notes in Computer Science, pages 121-136. Springer Berlin Heidelberg, Berlin, Heidelberg.

Martin D Sykora, Thomas W Jackson, and Suzanne Elayan. 2013. Emotive ontology: extracting finegrained emotions from terse, informal messages. IADIS International Journal on Computer Science and Information Systems, 8(2):106-118. Frederik Vaassen. 2014. Measuring emotion. Ph.D. thesis, Universiteit Antwerpen.

Xuren Wang and Qiuhui Zheng. 2013. Text Emotion Classification Research Based on Improved Latent Semantic Analysis Algorithm. In Proceedings of the 2nd International Conference on Computer Science nd Electronics Engineering (ICCSEE 2013), number Iccsee, pages 210-213, Paris, France. Atlantis Press.

Wenbo Wang, Lu Chen, Krishnaprasad Thirunarayan, and Amit P. Sheth. 2012. Harnessing Twitter "Big Data" for Automatic Emotion Identification. In 2012 International Conference on Privacy, Security,Risk and Trust and 2012 International Conference on Social Computing, pages 587-592. IEEE Computer Society, September. 